\title{
NONSTATIONARY STAGE OF QUASI-CHERENKOV BEAM INSTABILITY IN PERIODICAL STRUCTURES ${ }^{1}$
}

\author{
K. BATRAKOV and S. SYTOVA \\ Institute for Nuclear Problems, Belarussian State University \\ Bobruiskaya 11, 220050 Minsk, Belarus \\ E-mail: batrakov@inp.minsk.by; sytova@inp.minsk.by
}

Received October 1, 2004; revised January 3, 2005

\begin{abstract}
Analysis of nonstationary stage of quasi-Cherenkov instability of electron beam in the case of two-wave distributed feedback is carried out. Mathematical models and numerical methods of nonstationary quasi-Cherenkov electron beam instability are proposed. Results of numerical experiments are proposed. Bifurcations of nonstationary solution are discussed.

Key words: quasi-Cherenkov instability, numerical modelling, nonlinear integro-differential system, bifurcations
\end{abstract}

\section{Introduction}

This contribution is devoted to further analysis of nonstationary stage of quasiCherenkov instability of electron beam in Volume Free Electron Laser (VFEL) by methods of mathematical modelling.

Nowadays FEL (free electron laser) lasing is obtained in different wavelength ranges: from centimeter to ultraviolet. The high expensive international X-ray FEL project is on the preparation stage now [7]. VFEL based on mechanism of multiwave volume distributed feedback (VDFB) was proposed in [1, 4]. VFELs give possibility to reduce starting currents, to provide generation in large volume, to tune generation frequency [1]. Due to large electron beam cross section, VFEL generation in large volume essentially increases the electric strength of resonator and, in principle, allows to produce electromagnetic pulses of high power (greater than $10 \mathrm{GW}$ ). Besides the multi-wave distributed feedback VFEL provides the modes discrimination in the case when linear sizes of resonator (waveguide) cross section exceed generated wavelength ( in so-called oversized systems). First lasing of VFEL in millimeter range was recently obtained by a group of scientists from the Institute for Nuclear Problems [2].

\footnotetext{
${ }^{1}$ Authors thank prof. V. G. Baryshevsky for permanent interest to their work
} 
VFEL is a system with a great number of parameters. Varying these parameters causes changes in VFEL generation and transitions between different generation regimes, e.g. transition to the convective instability (amplification regime) and from the convective instability to absolute one (oscillator regime). Mentioned instabilities demonstrate also different features depending on parameters. There are stationary nonlinear oscillator regime and non-stationary oscillator regime in the case of absolute instability. Non-stationary regime is also divided into regimes with different periods of electron beam and radiation modulation and chaotic instability regime. Analysis of existing regimes, transitions between them (bifurcations) and parameters at which these transitions occur (bifurcation points and regions) is very important for description of VFEL operation. Quasi-Cherenkov (parametric) radiation is one of the possible mechanisms to realize VFEL generation [3]. In this case spatial-periodic system provides simultaneously Cherenkov synchronism condition and VDFB. Simulation of quasi-Cherenkov VFEL and its operation in different regimes is the aim of this paper.

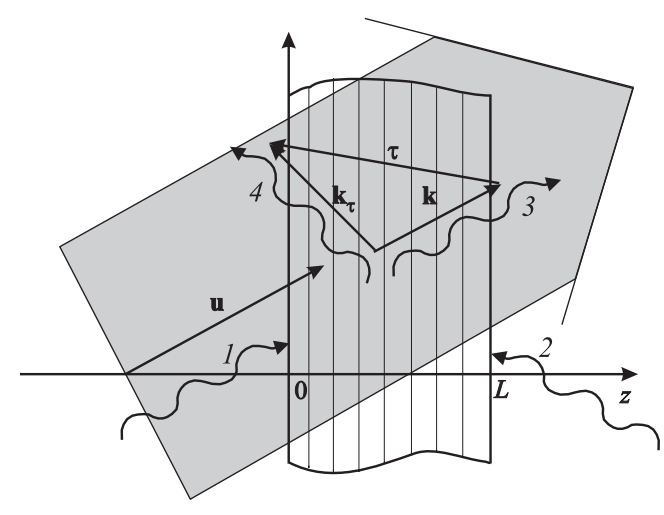

Figure 1. A simple scheme of quasi-Cherenkov VFEL (Bragg geometry).

\section{Mathematical Formulation}

In our previous paper [5] we presented a simple scheme of VFEL (see Fig. 1). An electron beam with electron velocity $\mathbf{u}$ passes through the target. This target of length $L$ is a spatially periodic medium. Incident electromagnetic waves 1 or 2 or 1 and 2 simultaneously emerge at the target. Waves 3 and 4 are two strong waves excited in the target, when specific conditions (so-called Bragg conditions) are fulfilled for generation of quasi-Cherenkov radiation. If electrons are under Cherenkov condition, they emit electromagnetic radiation in direction 3 or 4 or both directions depending on diffraction regime. A case without incident waves corresponds to oscillator generation regime. In Bragg geometry (Fig. 1) transmitted wave 3 and diffracted wave 4 are directed in opposite directions relative to axis $z$. In Laue geometry the waves propagate in the same direction. 
Let us consider the system of equations describing nonstationary quasi-Cherenkov instability. We write out it in a general form without accurate definition of coefficients.

$$
\begin{aligned}
& \frac{\partial E}{\partial t}+a_{1} \frac{\partial E}{\partial z}+b_{11} E+b_{12} E_{\tau} \\
& \quad=\Phi \int_{0}^{2 \pi} \frac{2 \pi-p}{8 \pi^{2}}(\exp (-i \Theta(t, z, p))+\exp (-i \Theta(t, z,-p))) d p \\
& E(t, 0)=E_{0}, \quad E(0, z)=0, \\
& \frac{\partial E_{\tau}}{\partial t}+a_{2} \frac{\partial E_{\tau}}{\partial z}+b_{21} E+b_{22} E_{\tau}=0 \\
& E_{\tau}(t, L)=E_{1}, \quad E_{\tau}(0, z)=0, \\
& \frac{\partial^{2} \Theta(t, z, p)}{\partial z^{2}}=\Psi\left(k-\frac{\partial \Theta(t, z, p)}{\partial z}\right)^{3} \operatorname{Re}(E(t-z / u, z) \exp (i \Theta(t, z, p))) \\
& \Theta(t, 0, p)=p, \quad \frac{\partial \Theta(t, 0, p)}{\partial z}=k-\frac{\omega}{u}
\end{aligned}
$$

where $i$ is the imaginary unit, $t>0, z \in[0, L], p \in[-2 \pi, 2 \pi]$.

$(2.1)-(2.3)$ is a system of integro-differential equations. In addition to temporal argument there are two independent arguments: spatial coordinate $z$ and initial electron phase $p$. Amplitudes of electromagnetic fields $E(t, z), E_{\tau}(t, z)$ and coefficients $a, b$ and $\Phi$ are complex-valued. Function $\Theta(t, z, p)$ is the phase of electron in electromagnetic wave. Function $\Theta$ and coefficient $\Psi$ are real. $k$ is a projection of wave vector on axis $z, \omega$ is a field frequency, $u$ is an initial electron beam velocity. Boundary conditions are written for the case of Bragg geometry.

Equation (2.3) describes a propagation of the electron beam in VFEL. We model it by averaging over initial phases of electrons. This method is well-known [12] and widely used in simulation of BWT (backward wave tube), TWB (traveling wave tube), FEL and other electronic devices. Next we present a derivation of (2.3). We consider a magnetized electron beam and assume that its propagation can be considered as one-dimensional. The motion equation of one electron in the wave has the following form:

$$
\ddot{z}=\frac{e}{m \gamma^{3}}\left(\vec{e}_{\sigma} \vec{n}\right) \operatorname{Re}\left\{a \exp \left(i \vec{k}_{\perp} \vec{r}_{\perp}+i k_{z} z-i \omega t\right)\right\},
$$

where $e$ and $m$ are electron charge and mass respectively, $\gamma$ is the Lorentz factor of electron beam. Initial phase is an individual mark of the electron in beam. Averaging over this phase allows us to pass from microscopical description to macroscopical one. Averaging current and applying Liuville's Theorem lead to the following equation:

$$
\ddot{z}=\frac{e n_{0} u}{2 \pi} \frac{1}{2 \pi} \int_{0}^{2 \pi} d \Theta_{1} \int_{\omega(t-z / u)}^{\omega(t-z / u)+2 \pi} d \Theta_{0} \exp \left\{-i \Theta\left(t, \Theta_{1}-\Theta_{0}\right)\right\},
$$


where $n_{0}$ is a beam density, $\Theta\left(t, t_{0}, \vec{r}_{\perp}\right)=k_{z} z+\vec{k}_{\perp} \vec{r}_{\perp}-\omega t\left(z, t_{0}\right)$ is an electron phase, $t\left(z, t_{0}\right)$ is a trajectory of electron emerged at moment $t_{0}$ in the target. Initial phase of the electron in interaction region has the form:

$$
\Theta\left(t=t_{0}, t_{0}, \vec{r}_{\perp}\right)=\vec{k}_{\perp} \vec{r}_{\perp}-\omega t_{0}=\Theta_{1}-\Theta_{0}
$$

Producing some transformations one can reduce the averaging over phases $\Theta_{0}$ and $\Theta_{1}$ to the averaging over one phase $p \in[-2 \pi, 2 \pi]$ and obtain equation (2.3).

In [5] we have considered the following stationary system of equations describing quasi-Cherenkov instability:

$$
\left\{\begin{array}{l}
\frac{d E}{d z}+a_{11} E+a_{12} E_{\tau}=\Phi \int_{0}^{2 \pi} \frac{2 \pi-p}{8 \pi^{2}} \\
\quad \times(\exp (-i \Theta(z, p))+\exp (-i \Theta(z,-p))) d p \\
\frac{d E_{\tau}}{d z}+a_{21} E+a_{22} E_{\tau}=0, \quad E(0)=E_{0}, \quad E_{\tau}(L)=E_{1}, \\
\frac{d^{2} \Theta(z, p)}{d z^{2}}=\Psi\left(k-\frac{d \Theta(z, p)}{d z}\right)^{3} \operatorname{Re}(E(z) \exp (i \Theta(z, p))), \\
\Theta(0, p)=p, \quad \frac{d \Theta(0, p)}{d z}=0 .
\end{array}\right.
$$

Now the nonstationary system $(2.1)-(2.3)$ is considered instead of (2.4). Different types of instabilities and bifurcations leading to transitions between these types of instabilities are studied on the basis of $(2.1)-(2.3)$.

\section{Numerical Algorithms}

We use notations from [11] and introduce in domain

$$
\Omega=\{0 \leq z \leq L, \quad-2 \pi \leq p \leq 2 \pi\} \times\{t>0\}
$$

uniform grids with respect to $t, z$ and $p$ :

$$
\begin{aligned}
& \omega_{t}=\left\{t_{l}=l h_{t}, l \geq 0\right\}, \omega_{z}=\left\{z_{m}=m h_{z}, m=0,1, \ldots, M, M h_{z}=L\right\}, \\
& \omega_{p}=\left\{p_{j}=j h_{p}, \quad j=-N, \ldots,-1,0,1, \ldots, N, h_{p} N=2 \pi\right\} .
\end{aligned}
$$

Discrete functions, defined on the grid, are denoted by

$$
\widehat{E}_{m}=E\left(t_{l+1}, z_{m}\right), \quad E_{m}=E\left(t_{l}, z_{m}\right), \quad \Theta_{m}^{j}=\Theta\left(t_{l}, z_{m}, p_{j}\right) .
$$

We approximate the differential problem (2.1) - (2.3) with the following finitedifference scheme:

$$
\widehat{\Theta}_{\bar{z} z}^{j}=\Psi\left(k-\widehat{\Theta}_{\stackrel{\circ}{j}}^{j}\right)^{3} \operatorname{Re}\left(\widetilde{E} \exp \left(i \widehat{\Theta}^{j}\right)\right), \quad j=0, \pm 1, \ldots, \pm N,
$$




$$
\begin{aligned}
& E_{t}+a_{1} \widehat{E}_{\bar{z}}+b_{11} \widehat{E}+b_{12} \widehat{E}_{\tau}=\Phi \sum_{j=0}^{N} c_{j}\left(\exp \left(-i \widehat{\Theta}^{j}\right)+\exp \left(-i \widehat{\Theta}^{-j}\right)\right), \\
& E_{\tau t}+a_{2} \widehat{E}_{\tau z}+b_{21} \widehat{E}+b_{22} \widehat{E}_{\tau}=0
\end{aligned}
$$

where $\widetilde{E}=E\left(t_{l}-\alpha t_{m}, z_{m}\right), \alpha=\left\lfloor h_{z} /\left(h_{t} u\right)\right\rfloor$. When $t_{l}-\alpha t_{m}<0$ we assume that $\widetilde{E}=E\left(0, z_{m}\right)$. Here $c_{j}$ are coefficients of the quadrature trapezoidal rule.

In [5] we have proposed the following iterative algorithm for solving (2.4):

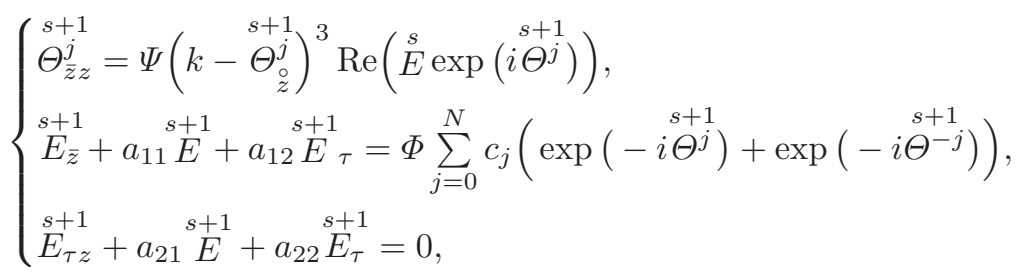

where $s \geq 0$ is a number of iteration, $\stackrel{0}{\Theta^{j}}=h_{p} j, \stackrel{0}{E}=0, \stackrel{0}{E}{ }_{\tau}=0$.

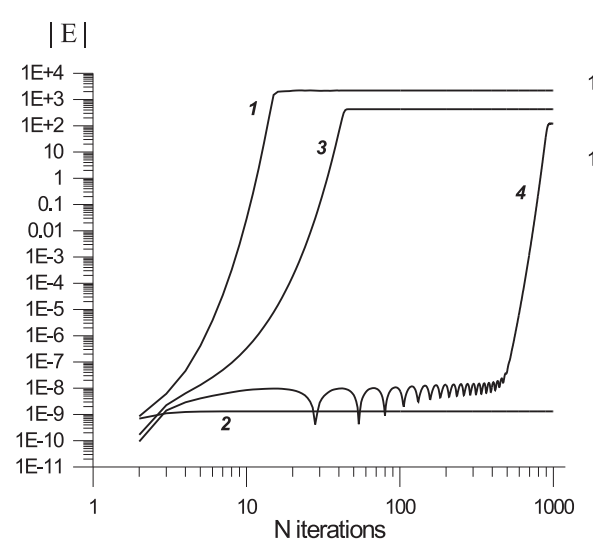

a

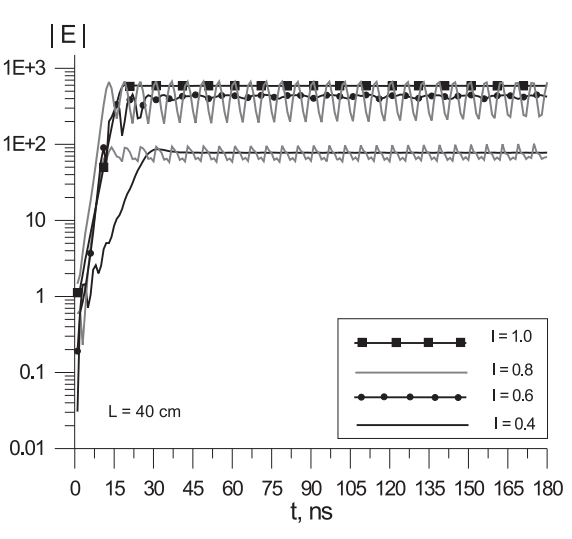

b

Figure 2. Numerical solutions: (a) stationary system, dependence on the number of iterations, (b) nonstationary system, dependence on $t$.

Let us demonstrate the dependence of stabilization of solution (3.2) on the number of iterations (see Fig. 2a). When the current density threshold is exceeded (curve 1 ) or there is no radiation amplification (curve 2), then about ten iterations are sufficient. For the other cases it is necessary to make $20-50$ iterations (curve 3). In the region near generation threshold, where solution is settled very slowly, it is necessary to compute a few hundreds of iterations (curve 4).

We are interested in investigation of dynamics of the whole system, since it is well-known that in laser systems different types of instabilities (bistability, pulsed solutions, chaos) can appear ([9], [10]). For different reasons namely lasers played a decisive role in development of synergetics. 
Problems of numerical stability of solutions of PDEs of a similar nature were investigated in [6]. But since it seems to be impossible to investigate analytically the stability of nonlinear integro-differential time-dependent problem (2.1) - (2.3), we restrict ourselves to numerical investigations. During computations we have changed different control parameters of the system (see Fig. 2b, Fig. 3-Fig. 5). There exist more than ten such parameters. As a result at all plots calculated for Bragg geometry we obtain Hopf's bifurcations $[8,9,10]$ which lead to transition between generation regimes with single or some basic frequencies or stable steady solutions.

If for some control parameters the solutions are unstable, then under random perturbations the trajectory sweeps chaotically in a large phase space. As a random perturbation we can consider computational errors in right-hand sides of the system. As a result we can obtain turbulence or chaos. In our experiments we get turbulence only in Laue geometry for large length of the target and high current density. In Bragg geometry we obtain typical self-oscillations with some principal frequencies. We suppose that for Laue geometry we obtain Hopf's bifurcations with wide set of principal frequencies too. This corresponds to numerous bifurcations of limit cycle to the torus.

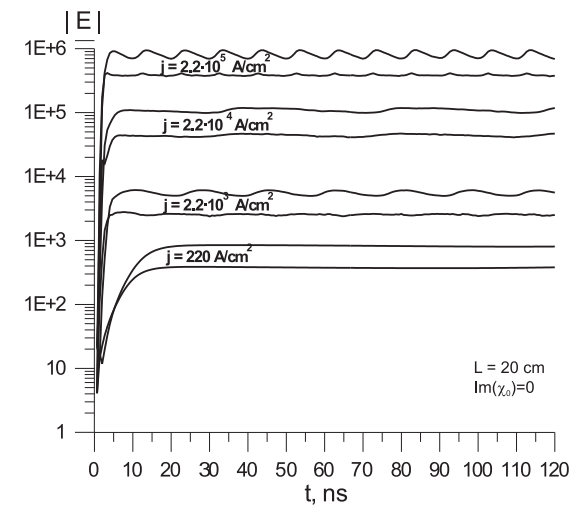

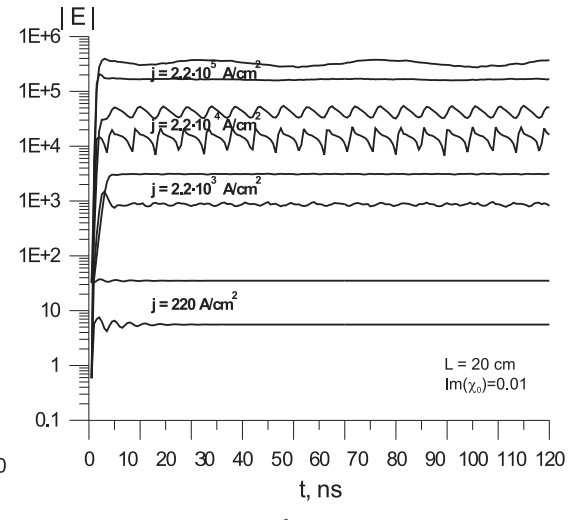

b

Figure 3. Numerical solution for different current density $j$ without (a) and with (b) absorption in Bragg geometry.

Since our numerical algorithms (3.1) and (3.2) are nonlinear, it seems to be impossible to investigate their convergence. If we consider linearized cases of these processes all becomes evident. So, according to numerical experiments, our schemes proved itself be stable and numerical solutions converged to analytical solutions of the initial differential system.

\section{Numerical Results for the Nonstationary System}

Let us discuss results of numerical experiments carried out. In this paper we want to give several examples of numerical calculations describing behavior of the solution 


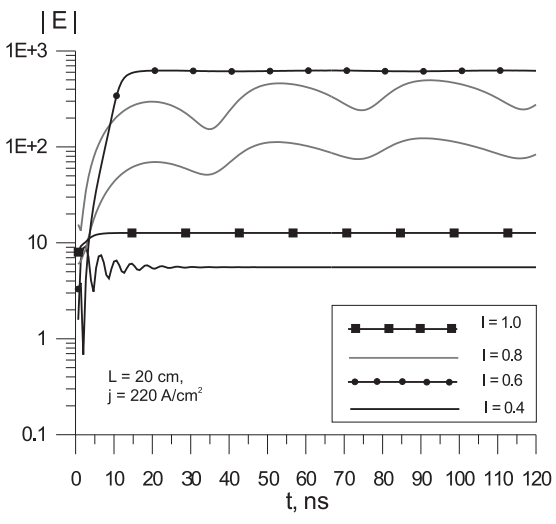

a

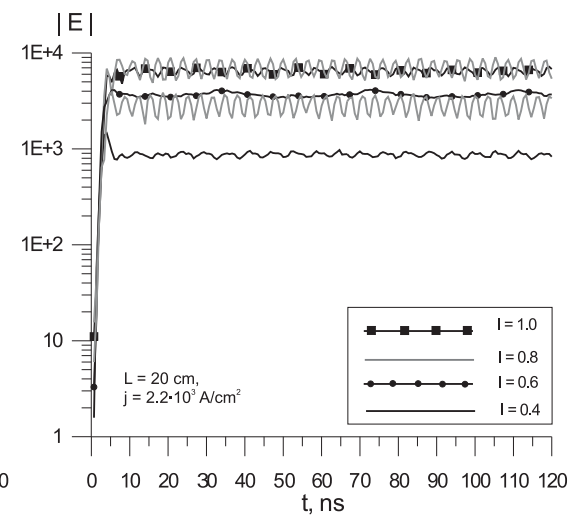

$\mathrm{b}$

Figure 4. Numerical solution for different detuning parameter $l$ in Bragg geometry.

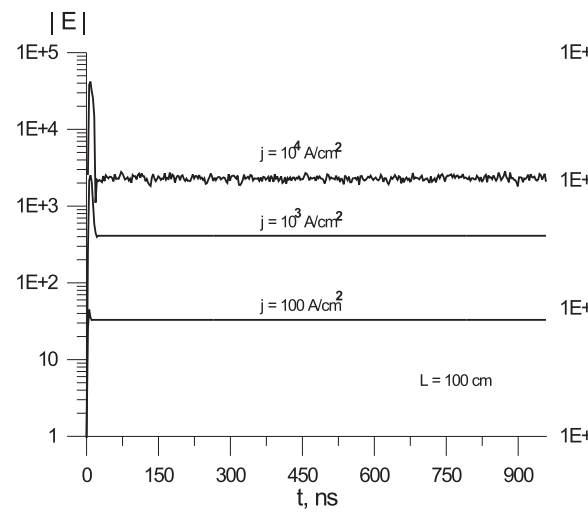

a

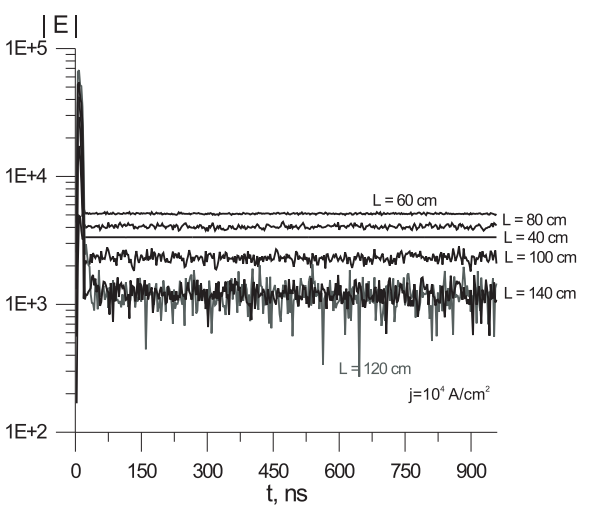

b

Figure 5. Numerical solution for different current density $j$ (a) and different length $L$ (b) in Laue geometry.

of nonstationary system. In each graphic plot only one control parameter is changed. That leads to transition between different regimes of generation in accordance with transition of parameter from one critical value to the other one. This parameter can be a beam current density $j$, detuning parameter $l$, absorption coefficient $\operatorname{Im}\left(\chi_{0}\right)$, target length $L$, frequency and so on. In Fig. 2b, Fig. 3-Fig. 5, one can see curves with single period of oscillations, with some basic frequencies and steady states. For example in Fig. 3 curves of amplitudes $|E(t, L)|$ (lower curve) and $\left|E_{\tau}(t, 0)\right|$ (upper curve in each pair) are presented. It is interesting that in each pair periods of oscillations of both curves are different. In Laue geometry (see Fig. 5) a turbulence induced by numerous bifurcations is obtained for large length of the target $L$ (greater than $80 \mathrm{~cm})$.

In two-wave Bragg diffraction geometry different generation regimes include also cases when one or two electromagnetic modes are in synchronism with elec- 
tron beam. During nonlinear dynamics this leads to transition between these modes and to more indicative bifurcation picture in Bragg diffraction geometry. More possible regimes exist for multi-wave diffraction which shall be considered in our future works.

\section{Conclusions}

The proposed mathematical models and numerical algorithms can be used effectively for modelling nonlinear regimes of VFEL operation. They will be useful for providing experiments on VFEL on the installation VFEL-300 keV created at the Institute for Nuclear Problems of Belarussian State University.

\section{References}

[1] V. Baryshevsky, K. Batrakov and I. Dubovskaya. Parametric (quasi-Cherenkov) X-ray FEL. Journ.Phys.D, 24, 1250 - 1257, 1991.

[2] V. Baryshevsky and K. Batrakov et al. First lasing of a volume FEL (VFEL) at a wavelength range 4-6 mm. Nucl. Instr. and Meth. in Phys. Res., A483, 21 - 24, 2002.

[3] V. Baryshevsky and I. Feranchuk. Parametric X-rays from ultrarelativistic electrons in a crystals: theory and possibilities of practical utilization. Journ.Phys., 44, 913 - 922, 1983.

[4] V. Baryshevsky and I. Feranchuk. Parametric beam instability of relativistic charged particles in a crystal. Phys.Let.A, 102, $141-144,1984$.

[5] K. Batrakov and S. Sytova. Modelling of quasi-Cherenkov electron beam instability in periodical structures. Mathematical Modelling and Analysis, 9(1), 1 - 8, 2004.

[6] Raim. Čiegis and Rem. Čiegis. Numerical stability analysis of solutions of PDEs. Computational Methods in Applied Mathematics, 4(1), 23 - 33, 2004.

[7] R. Brinkmann et al. TESLA XFEL, First Stage of the X-Ray Laser Laboratory. DESY Report 2002-167/TESLA-FEL 2002-09, Hamburg, 2002.

[8] G.Iooss and D. Joseph. Elementary stability and bifurcation theory. Springer-Verlag, Berlin, 1981.

[9] H. Haken. Advanced synergetics. Springer-Verlag, 1983.

[10] G. Nicolis and I.Prigogine. Exploring complexity. W.Y. Freeman and Company, New York, 1989.

[11] A.A. Samarskii. Theory of finite-difference schemes. Nauka, Moscow, 1989. (in Russian)

[12] L.A. Vainshtein and V.A. Solncev. Lectures on microwave electronics. Sov. Radio, Moscow, 1973. (in Russian)

\section{Nestacionariosios fazès kvazi-Cherenkovo spinduliuotės nestabilumas periodinèse struk-} tūrose

K. Batrakov, S. Sytova

Straipsnyje analizuojama nestacionariosios fazės kvazi-Cherenkovo elektronu spinduliuotès nestabilumas esant dviejų bangu sąveikai su grižtamo ryšio pernešimu. Pasiūlyti šios elektronų spinduliuotès nestacionariosios fazès atveju matematiniai ir skaitiniai sprendimo metodai. Pateikti skaitinio eksperimento rezultatai. Aptartos nestacionariojo sprendinio bifurkacijos. 